\title{
EXERCÍCIO FÍSICO NO TRATAMENTO DE FIBROSE DE CÍSTICA EM CRIANÇAS: UMA REVISÃO SISTEMÁTICA
}

\section{PHYSICAL EXERCISE IN THE TREATMENT OF CYSTIC FIBROSIS IN CHILDREN: A SYSTEMATIC REVIEW}

\author{
Eliane Mattana Griebler ${ }^{1}$, Maurício da Silva César ${ }^{1}$, \\ Débora Gaspary de Azeredo ${ }^{1}$, Paulo José Cauduro Marostica ${ }^{2,3}$, \\ Ângela d'Avila Harthmann ${ }^{4}$
}

\begin{abstract}
RESUMO
Evidências mostram que o exercício físico gera efeito positivos em indivíduos com fibrose cística, melhorando o prognóstico, a qualidade de vida e reduzindo o número de internações. O estudo se caracteriza como qualitativo e de revisão sistemática. A partir dos descritores "fibrose cística", "criança" e "exercício físico" realizou-se uma busca por artigos nas bases de dados do Portal de Periódicos da CAPES e Medline/PubMed. Desta busca originaram-se inicialmente 125 artigos. Após refinamento, foram selecionados 14 artigos que se adequaram aos critérios propostos pela pesquisa. Utilizou-se como critérios de inclusão a leitura prévia dos resumos, nos quais deveriam conter informações referentes à utilização do exercício físico como tratamento da fibrose cística, ser um estudo com população alvo $\leq 20$ anos de idade e que utilizassem protocolo validado de exercícios físicos. Os artigos encontrados trazem relatos dos benefícios da prática regular de exercícios físicos no tratamento efetivo de pacientes com fibrose cística na infância e adolescência. Os achados são referentes à melhora da capacidade cardiorrespiratória, da postura, além de outros benefícios como aumentos na autoestima e na qualidade de vida destes indivíduos. Grande parte dos trabalhos avaliam o efeito do exercício físico a curto prazo, demonstrando que os dados oriundos dos estudos atuais são escassos e que são necessários mais estudos para maior esclarecimento do tema.
\end{abstract}

Palavras-chave: Fibrose cística; criança; exercício físico

\section{ABSTRACT}

Evidence shows that physical exercise produces positive effects on individuals with cystic fibrosis, improving their prognosis and quality of life and reducing the number of hospitalizations. This study was qualitative and consisted of a systematic review. Using the descriptors "cystic fibrosis," "child" and "physical exercise," a search for articles was carried out in the databases of CAPES Journals Portal and MEDLINE/PubMed. This initial search yielded 125 articles. After narrowing, 14 articles meeting the study criteria were selected. Abstracts were screened in search of the following inclusion criteria: having information on the use of physical exercise as treatment of cystic fibrosis, having a target population aged $\leq 20$ years, and using a validated exercise protocol. The articles report the benefits of regular physical exercise in the effective treatment of patients with cystic fibrosis in childhood and adolescence. The findings show improved cardiorespiratory capacity and posture, as well as other benefits such as increased self-esteem and quality of life of these individuals. Most studies evaluated short-term effects of physical exercise, demonstrating that current data are still incipient and further studies are needed to investigate the subject.

Keywords: Cystic fibrosis; child; physical exercise

A fibrose cística (FC) é uma doença genética, de herança autossômica recessiva, de evolução crônica e progressiva, caracterizada por doença pulmonar crônica, insuficiência pancreática e níveis elevados de eletrólitos no suor ${ }^{1-3}$. É causada por mutações em um único gene no braço longo do
Clin Biomed Res. 2019;39(1):69-74

1 Programa de Residência Integrada Multiprofissional em Saúde, Saúde da Criança, Hospital de Clínicas de Porto Alegre (HCPA). Porto Alegre, RS, Brasil.

2 Departamento de Pediatria, Universidade Federal do Rio Grande do Sul (UFRGS), Hospital de Clínicas de Porto Alegre (HCPA). Porto Alegre, RS, Brasil

3 Unidade de Pneumologia Infantil do Hospital de Clínicas de Porto Alegre (HCPA). Porto Alegre, RS, Brasil

4 Serviço de Educação Física e Terapia Ocupacional, Hospital de Clínicas de Porto Alegre (HCPA). Porto Alegre, RS, Brasil.

Autor correspondente: Ângela d'Avila Harthmann aharthmann@hcpa.edu.br Hospital de Clínicas de Porto Alegre Rua Ramiro Barcelos, 2350. 90035-903, Porto Alegre, RS, Brasil. 
cromossomo 7 que codifica a proteína reguladora da condutância transmembrana ou Cystic Fibrosis Transmembrane Condutance Regulator (CFTR) ${ }^{4}$. A doença é mais frequente em indivíduos caucasianos e ocorre em aproximadamente 1:2.500 recém-nascidos vivos no mundo ${ }^{5}$. No Brasil, a incidência varia de acordo com a região, sendo que a região sul do país apresenta incidência similar à população caucasiana centro-europeia (1/2.000 e 1/5.000 nascidos vivos), enquanto nas outras regiões esta estimativa se reduz para $1 / 10.000$ nascidos vivos ${ }^{6,7}$.

A FC leva a alterações patológicas de órgãos que expressam a proteína CFTR nas células epiteliais, especificamente as vias respiratórias (incluindo os seios nasais e os pulmões), o trato gastrointestinal (incluindo o pâncreas e o sistema biliar), as glândulas sudoríparas e o sistema genitourinário ${ }^{8}$. A doença é diagnosticada pela presença de pelo menos um achado fenotípico ou história familiar de irmão com FC ou triagem neonatal positiva, acompanhados de evidência laboratorial de disfunção da CFTR. O método padrão ouro para o diagnóstico da FC é realizado através da dosagem de eletrólitos no suor ${ }^{9}$.

O tratamento padrão envolve antibioticoterapia, higiene das vias aéreas, exercício físico, mucolíticos, broncodilatadores, oxigênio, agentes anti-inflamatórios e suporte nutricional ${ }^{10}$. O exercício físico quando associado ao tratamento melhora a capacidade física de crianças e jovens com FC ${ }^{11}$. Evidências mostram que o exercício físico associado à terapia padrão em paciente com FC melhora a capacidade funcional e estado nutricional, aumenta a depuração mucociliar e a densidade mineral óssea e minimiza o declínio da função pulmonar, melhorando o prognóstico e a qualidade de vida e reduzindo o número de internações ${ }^{12-16}$. Em comparação com indivíduos saudáveis, as crianças com FC demonstram diminuição da função ventilatória e desnutrição, propiciando a fadiga muscular e prejudicando o desempenho e a capacidade ao exercício ${ }^{17,18}$. Sendo assim, a prática de atividade física regular é de extrema importância no tratamento de pacientes com FC e deve ser estimulada durante todo o processo de crescimento e desenvolvimento para que esse comportamento tenha maiores chances de ser transferido para a vida adulta ${ }^{13,19}$. A partir disto, tem-se como objetivo analisar, através de revisão sistemática, os efeitos do exercício físico no tratamento da fibrose cística relatados na literatura em crianças e adolescentes.

\section{MÉTODOS}

\section{Tipo de Estudo}

O estudo se caracteriza como qualitativo e de revisão sistemática. Dentre os estudos que utilizam fontes de informações bibliográficas para obtenção de resultados de pesquisa de outros autores, buscando fundamentar teoricamente um determinado tema, encontram-se os artigos de revisão de literatura. Estes estudos podem ser categorizados como sendo de revisão narrativa e de revisão bibliográfica sistemática em função de terem objetivos e características distintos $^{20}$

Através desta revisão sistemática, verificamos estudos que respondam a seguinte questão: "os efeitos do exercício físico no tratamento da fibrose cística em crianças de zero até 19 anos de idade".

Como critério de inclusão optou-se por estudos que utilizaram programas de exercício físico no tratamento de crianças com FC, sendo eles randomizados ou não. Como critérios de exclusão, determinamos não inserir estudos observacionais e/ou de questionários via telefone.

\section{Busca nas Bases de Dados}

A partir dos descritores "fibrose cística", "criança" e "exercício físico" realizou-se uma busca por artigos nas bases de dados do Portal de Periódicos da CAPES e Medline/PubMed. Foram identificados inicialmente 125 artigos nas pesquisas nas bases de dados, 42 artigos em duplicata foram removidos posteriormente dentre os 83 artigos selecionados 69 foram excluídos após a leitura dos resumos e títulos. Então 14 artigos foram avaliados quanto a sua elegibilidade e 10 foram excluídos por não falarem diretamente sobre o tema da pesquisa, restando ao final 4 artigos para análise.

Utilizou-se como critérios de seleção a leitura prévia dos resumos, nos quais deveriam conter informações referentes à utilização do exercício físico como tratamento da fibrose cística, ser um estudo com população alvo $<20$ anos de idade e que utilizassem protocolo validado de exercícios físicos. Apesar de utilizar-se o descritor "criança" definiu-se esse limiar de idade por ser o que se apresentou na literatura após a busca. A figura 1 mostra de forma detalhada o processo de seleção dos artigos. Mesmo sem inserir o termo adolescente nos descritores da pesquisa foram poucos estudos encontrados e dentre eles a população foi descrita como jovem incluindo os adolescentes, e por este motivo deixamos a média de idade de acordo com os artigos encontrados.

\section{RESULTADOS E DISCUSSÃO}

$\mathrm{Na}$ revisão sistemática foram encontrados ensaios clínicos randomizados e ensaios clínicos não randomizados. Dentre os objetivos destes estudos, podemos citar: comparar o treinamento aeróbico e de resistência ${ }^{21}$, avaliar se a atividade física habitual vigorosa regular pode prevenir a deterioração do 


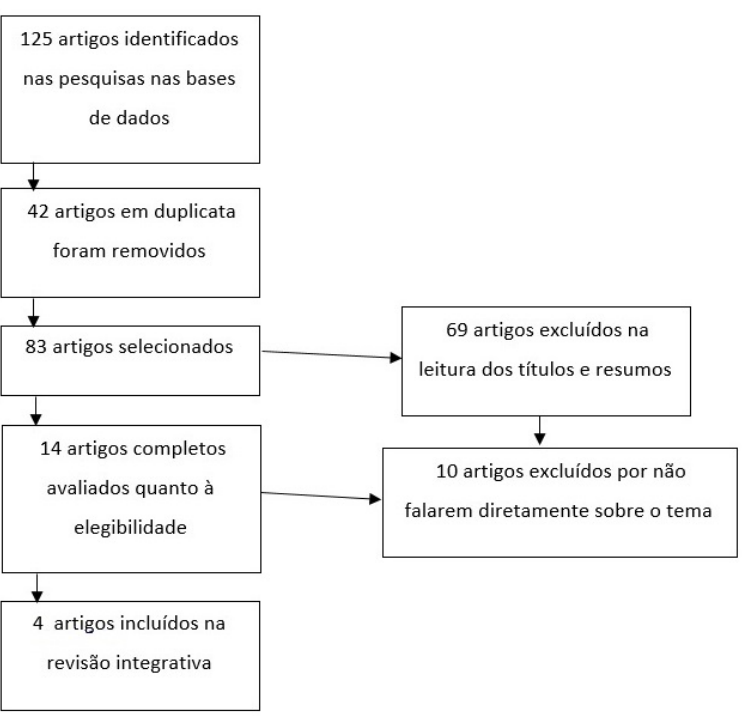

Figura 1: Fluxograma referente à metodologia de seleção dos artigos.

declínio do volume expiratório forçado no primeiro segundo (VEF1) ao longo do tempo 22 , verificar a resposta do treinamento físico associada à inflamação e ao status de infecção ${ }^{23}$ e avaliar os efeitos de um treinamento aeróbico máximo e submáximo ${ }^{24}$.

De forma geral, os estudos analisados demonstraram benefícios do exercício físico em crianças e adolescentes com FC, principalmente em variáveis como a função pulmonar, o efeito do exercício de força, aeróbico e de resistência muscular.

Algumas das modalidades utilizadas foram a corrida, a caminhada e o ciclismo. A capacidade física foi medida pelo teste de exercício em esteira incremental ${ }^{21}$, e com testes de exercício em cicloergômetro com carga progressiva ${ }^{22-24}$. Constatamos grande variação em relação a frequência semanal, assim como o tempo de duração das atividades físicas nos protocolo de intervenção dos artigos selecionados.

O resumo das características e resultados dos estudos é apresentado na tabela 1.

Todos os estudos analisados utilizaram atividades aeróbicas, sendo que três artigos associaram atividades aeróbicas e de resistência ${ }^{21-23}$, e um artigo comparou o treinamento aeróbico e de resistência ${ }^{21}$. Em apenas um dos estudos ${ }^{22}$, as atividades foram desenvolvidas de maneira parcialmente supervisionada. Por se tratar de um estudo clínico randomizado controlado multicêntrico internacional, aplicou-se um protocolo próprio que intercala atividades de fortalecimento muscular e exercícios aeróbicos.

Nesta revisão foram excluídos todos os estudos observacionais, evitando assim, riscos de viés. Dentre os estudos incluídos, foi encontrado um estudo com supervisão parcial de programa de exercícios físicos entretanto, apesar dessa característica, é um estudo clínico randomizado controlado multicêntrico internacional. Pela sua relevância, foi inserido nesta revisão. Evidenciou-se neste caso que somente 1 dos estudos é randomizado, e que de maneira geral os estudos incluídos contam com um $\mathrm{n}$ pequeno, sem maiores implicações em decorrência disto.

Corroborando com nossos achados, a literatura sugere que o melhor programa de treinamento possa ser composto por uma combinação de treinamento aeróbico e de resistência. Esta combinação de exercícios aeróbicos e de treinamento de força tem impacto positivo sobre a função pulmonar em pacientes com $\mathrm{FC}$, bem como diminuem a queda progressiva da função pulmonar, aumentam a resistência ao exercício e melhoram a autoestima e a qualidade de vida dos pacientes com $\mathrm{FC}^{25-27}$ encontraram em seu estudo que ambos tipos de treinamento podem aumentar a capacidade física de crianças com FC, além de aumentar a força da parte superior do corpo. A combinação de treinamento aeróbico e treinamento de força se mostra segura e de boa tolerância, mesmo para pacientes com VEF1 $<80 \%$.

Selvadurai et al. ${ }^{21}$, compararam o efeito do treinamento aeróbico e de resistência em 67 crianças com FC, entre 8 a 16 anos de idade, demonstrando que o treinamento aeróbico aumenta significativamente a capacidade aeróbica de pico, níveis de atividade e qualidade de vida em relação às crianças que realizaram o programa de treinamento de resistência. As crianças que receberam treinamento de resistência apresentaram maior ganho de peso (massa total, bem como massa livre de gordura), melhora da função pulmonar e aumento de força em membros inferiores, quando comparadas com as que receberam treinamento aeróbico.

Sabe-se que a função pulmonar desses indivíduos geralmente é bastante comprometida. Dentre as variáveis utilizadas para a mensuração da funcionalidade respiratória, neste caso estão, a capacidade vital forçada, o volume expiratório forçado em 1 segundo e o fluxo expiratório forçado na metade média da CVF, que são medidos através da espirometria. O VEF1 é a variável que melhor reflete o estado da função pulmonar ao longo do curso da doença pulmonar por FC. Sendo também um indicador do prognóstico de sobrevida desses pacientes. Pacientes com VEF1 acima dos $55 \%$ do previsto estão aptos para a prática de exercícios físicos similares àqueles praticados por indivíduos saudáveis ${ }^{28,29}$. Medidas de função pulmonar são usadas como medida de desfecho em pacientes com FC, uma vez que existe uma forte associação 
Tabela 1: Síntese dos programas de exercícios para fibrose Cística, definidos a partir da estratégia de busca.

\begin{tabular}{|c|c|c|c|c|c|}
\hline Autor & Ano & $\begin{array}{c}\text { País } \\
\text { (Origem) }\end{array}$ & Modalidade exercício & Frequência e duração & $\begin{array}{l}\text { Principais } \\
\text { resultados }\end{array}$ \\
\hline Selvadurai et al & 2002 & Austrália & $\begin{array}{l}\text { Atividades aeróbicas, } \\
\text { treinamento resistido }\end{array}$ & 5 Sessões/30 min & $\uparrow C A, \uparrow N A F, \uparrow Q V$ \\
\hline Hebestreit et al & 2018 & Multicêntrico & $\begin{array}{l}\text { Atividades de fortalecimento } \\
\text { muscular, exercícios } \\
\text { aeróbicos }\end{array}$ & $\begin{array}{l}3 \text { horas por semana/ } \\
12 \text { meses }\end{array}$ & $\uparrow Q V, V F_{1}$ \\
\hline Pauline. B et al & 2013 & Holanda & $\begin{array}{l}\text { alongamento, abdominal, } \\
\text { flexões, extensão posterior e } \\
\text { o último a corrida. }\end{array}$ & $\begin{array}{l}5 \text { dias na semana / } \\
\text { por } 11 \text { min } \\
12 \text { semanas }\end{array}$ & $\uparrow C E$, RNAII \\
\hline Bragion et al & 1989 & Itália & $\begin{array}{l}\text { Corrida de resistência e com } \\
\text { obstáculo }\end{array}$ & $\begin{array}{l}3 x \text { por semana/ } 1 \\
\text { hora } \\
8 \text { semanas }\end{array}$ & $\uparrow R T M, S M$ \\
\hline
\end{tabular}

CA: capacidade aeróbica: NAF: níveis de atividade física, QV: qualidade de vida, CE: capacidade de exercício, RNAll: resposta negativa associada à inflamação e ao status de infecção, RTM: resposta ao treinamento máximo, SM: resposta ao treinamento submáximo, VEF $_{1}$ : volume de expiração máxima em 1 minuto.

entre função pulmonar e mortalidade ${ }^{30}$. Ao passo que pode se apresentar como um elemento importante na adesão ao tratamento, o comprometimento com o cuidado diário na FC é uma questão complexa ${ }^{21}$, principalmente nas crianças e adolescentes.

Uma questão importante de ser discutida sobre a população jovem com FC está no fato de, pelo pouco tempo de exposição a doença em relação a população adulta, a deterioração pulmonar ainda não estar tão agravada. E com a adesão precoce de programas de atividade física deixar esse processo cada vez mais tardio. Dessa forma, a prática regular de atividades físicas, bem como de programas de exercícios físicos tem efeito positivo na qualidade de vida dessa população.

Dois dos artigos ${ }^{21,23}$ resultantes da busca, mostram que a prática regular de atividades físicas melhora significativamente a função pulmonar. Este dado, é extremamente relevante, pois sabe-se que pacientes com FC frequentemente apresentam progressiva limitação ao exercício físico e redução de suas atividades de vida diária. As causas principais da intolerância ao exercício estão associadas à redução na capacidade ventilatória e da reserva ventilatória, perda da massa muscular esquelética periférica e diminuição da função cardiovascular. No entanto, pacientes com $\mathrm{FC}$ que praticam atividade física apresentam redução no declínio da função pulmonar e melhora da depuração das vias aéreas ${ }^{31}$.
Através desta revisão, foi possível verificar estudos com diferentes protocolos de intervenção. Além disso, apenas dois estudos realizaram ensaios clínicos randomizados, evidenciando a escassez de dados científicos mais rigorosos, que comprovem a relevância do exercício físico como parte do tratamento da FC em crianças e adolescentes.

\section{CONSIDERAÇÕES FINAIS}

Sendo assim, podemos afirmar que os efeitos do exercício físico em crianças e adolescentes com FC relatados na literatura são benéficos. Porém, os estudos analisados apresentam características muito heterogêneas, tanto na modalidade e frequência de exercícios, quanto nos indicadores avaliados.

Ressalta-se a relevância deste estudo, visto que a temática proposta ainda é pouco difundida e poucas são as pesquisas publicadas sobre o tema. Também salienta-se a falta de um protocolo de exercícios padrão e que avalie os efeitos da prática regular de exercícios físicos à longo prazo nessa população, caracterizando uma limitação do estudo.

\section{Conflito de Interesse}

Os autores declaram não haver conflitos de interesse. Com relação aos papéis de cada autor, os três primeiros autores realizaram a procura por artigos, enquanto os dois últimos foram supervisores do estudo. 


\section{REFERÊNCIAS}

1. Castellani C, Macek M JR Cassiman JJ, Duff A, Massie J, ten Kate LP, et al. Benchmarks for cystic fibrosis carrier screening: a European consensus document. $J$ Cyst Fibros. 2010;9(3):165-78. http:// dx.doi.org/10.1016/j.jcf.2010.02.005. PMid:20363197.

2. Daftary A, Acton J, Heubi J, Amin R. Fecal elastase-1: Utility in pancreatic function in cystic fibrosis. $J$ Cyst Fibros. 2006;5(2):71-6. http://dx.doi. org/10.1016/j.jcf.2006.01.005. PMid:16603421.

3. Kerem E, et al. Standars of care for patients with cystic fibrosis: a European consensus. J Cyst Fibros. 2005;4(7):7-21. http://dx.doi. org/10.1016/j.jcf.2004.12.002. PMid:15752677.

4. Gibson RL, Burns JL, Ramsey BW. Pathophysiology and management of pulmonary infections in cystic fibrosis. Am J Respir Crit Care Med. 2003;168(8):918-51. http://dx.doi. org/10.1164/rccm.200304-505SO. PMid: 14555458.

5. Chaves, CRMM. et al. Exercício aeróbico, treinamento de força muscular e testes de aptidão física para adolescentes com fibrose cística: revisão da literatura. Rev Bras Saude Mater Infant. 2007;7(3):245-50.

6. Ribeiro, JD; Ribeiro M; Ribeiro, AF. Controvérsias na fibrose cística - do pediatra ao especialista. $J$ Pediatr. 2000;78(2):171-86.

7. Raskin $S$, Pereira-Ferrari L, Reis FC, Abreu F, Marostica P, Rozov $\mathrm{T}$, et al. Incidence of cystic fibrosis in five different states of Brazilians determined by screening of $\mathrm{p}$. F508 del, mutation at the CFTR gene in newborns and patients. J Cyst Fibros. 2008;7(1):5-22. http://dx.doi. org/10.1016/j.jcf.2007.03.006. PMid:17544945.

8. Huffmyer JL, Littlewood KE, Nemergut EC. Perioperative management of the adult with cystic fibrosis. Anesth Analg. 2009;109(6):1949-61. http://dx.doi. org/10.1213/ANE.0b013e3181b845d0. PMid:19923526.

9. Adde FV. Fibrose cística na clínica pediátrica. Pediatr Mod. 2014;50(1):1-1.
10. Dalcin PTR, Abreu e Silva FA. Fibrose cística no adulto: aspectos diagnósticos e terapêuticos. J Bras Pneumol. 2008;34(2):107-117.

11. Lai HJ, Cheng $\mathrm{Y}, \mathrm{Cho} \mathrm{H}$, Kosorok MR, Farrell PM. Association between initial disease presentation, lung disease outcomes and survival in patients with cystic fibrosis. Am J Epidemiol. 2004;159(6):537-46. http://dx.doi.org/10.1093/aje/kwh083. PMid:15003957.

12. Baker CF, Wideman L. Attitudes toward physical activity in adolescents with cystic fibrosis: sex differences after training: a pilot study. $J$ Pediatr Nurs. 2006;21(3):197210. http://dx.doi.org/10.1016/j. pedn.2005.07.011. PMid:16713510.

13. Schneiderman-Walker J, Pollock SL, Corey M, Wilkes DD, Canny GJ, Pedder L, et al. A randomized controlled trial of a 3-year home exercise program in cystic fibrosis. $J$ Pediatr. 2000;136(3):304-10. http:// dx.doi.org/10.1067/mpd.2000.103408. PMid:10700685.

14. Zach MS, Oberwaldner B. Chest physiotherapy-the mechanical approach to anti-infective therapy in cystic fibrosis. Infection. 1987;15(5):381-4. http://dx.doi. org/10.1007/BF01647750. PMid:3319913.

15. Selvadurai HC, Blimkie CJ, Cooper PJ, Mellis CM, Van Asperen PP. Gender differences in habitual activity in children with cystic fibrosis. Arch Dis Child. 2004;89(10):928-33. http:// dx.doi.org/10.1136/adc.2003.034249. PMid:15383436.

16. Wilkes D, Schneider-Walker J, Corey $M$. Long term effect of habitual physical activity on lung health in patients with cystic fibrosis. Pediatr Pulmonol. 2007;42(30):358-9.

17. Pouliou E, Nanas $S$, Papamichalopoulos A, Kyprianou T, Perpati G, Mavrou I, et al. Prolonged oxygen kinects during early recovery from maximal exercise in adult patients with cystic fibrosis. Chest. 2001;119(4):1073-8. http://dx.doi. org/10.1378/chest.119.4.1073. PMid:11296172.

18. Shah AR, Gozal D, Keens TG. Determinants of aerobic and anaerobic execise performance in cystic fibrosis. Am J Respir Crit Care Med. 1998;157(4):114550. http://dx.doi.org/10.1164/ ajrccm.157.4.9705023. PMid:9563732.

19. Nixon PA, Orenstein DM, Kelsey SF, Doershuk CF. The prognostic value of exercise testing in patients with cystic fibrosis. N Engl J Med. 1992;327(25):1785-8. http://dx.doi. org/10.1056/NEJM199212173272504. PMid:1435933.

20. Botelho LLR, Cunha CCA, Macedo M. O método da revisão integrativa nos estudos organizacionais. Gest Soc. 2011;5(11):121-36. http://dx.doi. org/10.21171/ges.v5i11.1220.

21. Selvadurai HC, Blimkie CJ, Meyers N, Mellis CM, Cooper PJ, Van Asperen PP. Randomized controlled study of in-hospital exercise training programs in children with cystic fibrosis. Pediatr Pulmonol. 2002;33(3):194-200. http:// dx.doi.org/10.1002/ppul.10015. PMid:11836799.

22. Hebestreit $\mathrm{H}$, Lands LC, Alarie N, Schaeff J, Karila C, Orenstein $\mathrm{DM}$, et al. Effects of a partially supervised conditioning programme in cystic fibrosis: An International Multi-Centre Randomised Controlled Trial (ACTIVATE-CF): Study Protocol. BMC Pulm Med. 2018;18(1):31. http:// dx.doi.org/10.1186/s12890-018-05966. PMid:29422091.

23. van de Weert-van Leeuwen PB, Hulzebos HJ, Werkman MS, Michel $S$, Vijftigschild LA, van Meegen MA, et al. Chronic inflammation and infection associate with a lower exercise training response in cystic fibrosis adolescents. Respir Med. 2014;108(3):445-52. http://dx.doi. org/10.1016/j.rmed.2013.08.012. PMid:24480322.

24. Braggion $C$, Cornacchia M, Miano A, Schena F, Verlato G, Mastellay G. Exercise tolerance and effects of training in young patients with cystic fibrosis and mild airway obstruction. Pediatr Pulmonol. 1989;7(3):14552. http://dx.doi.org/10.1002/ ppul.1950070306. PMid:2797928.

25. van Doorn N. Exercise programs for children with cystic fibrosis: a systematic review of randomized controlled trials. Disabil Rehabil. 2010;32(1):41-9. http://dx.doi. org/10.3109/09638280902991842 PMid:19925275. 
26. Schindel CS, Donadio MVF. Efeitos de programas de exercício físico em pacientes com fibrose cística. Sci. med. 2013;23(3)

27. Orenstein DM, Hovell MF, Mulvihill M, Keating KK, Hofstetter CR, Kelsey $S$, et al. Strength vs. aerobic training in children with cystic fibrosis: a randomized controlled trial. Chest. 2004;126(4):1204-14. http://dx.doi. org/10.1378/chest.126.4.1204. PMid:15486384.
28. Turcios NL. Cystic fibrosis: an overview. J Clin Gastroenterol. 2005;39(4):307-17. http:// dx.doi.org/10.1097/01. mcg.0000155140.63510.cd. PMid:15758625.

29. Connett GJ. Respiratory care. In: Peebles A, Connett GJ, Maddison JC, Gavin J. Cystic fibrosis care: A practical guide. London: Churchill Livingstone; 2005. p. 37-57.
30. Morrow, BM. et al. Improvements in lung function of a pediatric cystic fibrosis population in a developing country. J. Pediatr. 2008;84(5):403-09.

31. Hebestreit H, Schmid K, Kieser $S$, Junge S, Ballmann M, Roth $\mathrm{K}$, et al. Quality of life is associated with physical activity and fitness in cystic fibrosis. BMC Pulm Med. 2014;14(1):26. http://dx.doi. org/10.1186/1471-2466-14-26. PMid:24571729.

Recebido: 19 ago, 2018 Aceito: 18 mar, 2019 